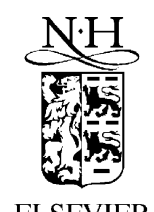

Information Processing Letters 76 (2000) 113-119

\title{
Information
} Processing

Letters

ELSEVIER

\section{On the number of spanning trees of a multi-complete/star related graph}

\author{
Kuo-Liang Chung a,*, Wen-Ming Yan ${ }^{\mathrm{b}, 1}$ \\ ${ }^{a}$ Department of Information Management, Institute of Computer Science \& Information Engineering, \\ National Taiwan University of Science and Technology, No. 43, Section 4, Keelung Road, Taipei 10672, Taiwan \\ ${ }^{\mathrm{b}}$ Department of Computer Science and Information Engineering, National Taiwan University, \\ No. 1, Section 4, Roosevelt Road, Taipei 10764, Taiwan
}

Received 14 June 1999; received in revised form 13 July 2000

Communicated by L.A. Hemaspaandra

\begin{abstract}
This paper derives a closed formula for the number of spanning trees of a multi-complete/star related graph $G=K_{n}-$ $K_{m}\left(a_{1}, a_{2}, \ldots, a_{l} ; b_{1}, b_{2}, \ldots, b_{m-l}\right)$, where $K_{m}\left(a_{1}, a_{2}, \ldots, a_{l} ; b_{1}, b_{2}, \ldots, b_{m-l}\right)$ consists of $l$ complete graphs and $m-l$ star graphs such that the $i$ th complete graph has $a_{i}+1$ nodes; the $j$ th star graph has $b_{j}+1$ nodes, and further, the related $m$ roots are connected together to form a complete graph. The proposed results extend previous results to a larger graph class. In addition, we provide a general maximization theorem for the multi-star graph. (c) 2000 Elsevier Science B.V. All rights reserved.
\end{abstract}

Keywords: Counting; Maximization; Spanning trees; Combinatorial problems

\section{Introduction}

An undirected simple graph $G$ consists of a set $V(G)$ of vertices and a set $E(G)$ of edges. A complete graph $K_{n}$ with $n$ vertices has one edge between each pair of distinct vertices. The complement $\bar{G}$ of a graph $G=(V, E)$ on $n$ vertices is defined to be the $n$ vertex graph containing exactly the edges of $K_{n}$ which are not in $G$. A multi-complete/star related (MCSR) graph,

$G=K_{n}-K_{m}\left(a_{1}, a_{2}, \ldots, a_{l} ; b_{1}, \ldots, b_{m-l}\right)$,

\footnotetext{
* Corresponding author. Supported by NSC89-2213-E011-061.

E-mail addresses: klchung@cs.ntust.edu.tw (K.-L. Chung), ganboon@csie.ntu.edu.tw (W.-M. Yan).

${ }^{1}$ Supported by NSC87-2119-M002-006.
}

is an $n$-vertex graph whose complement consists of $K_{m}$ with $l$ complete graphs and $m-l$ star graphs such that the $i$ th complete graph has $a_{i}+1$ nodes; the $j$ th star graph has $b_{j}+1$ nodes. In addition, the $m$ roots $(m-l$ roots come from the $m-l$ star graphs and $l$ roots come from the $l$ complete graphs, any one node in the complete graph being selected as the root) are connected together to form a complete graph; the remaining

$k=n-m-\sum_{i=1}^{l} a_{i}+\sum_{j=1}^{m-l} b_{j}$

vertices are isolated points. Fig. 1 illustrates the graph $K_{3}(3 ; 2,3)$.

Some special graphs considered in $[1,2,4,5]$ are covered in the MCSR graph mentioned above. Specifically, when setting $l=0$, i.e., $G=K_{n}-K_{m}\left(b_{1}, b_{2}\right.$, 


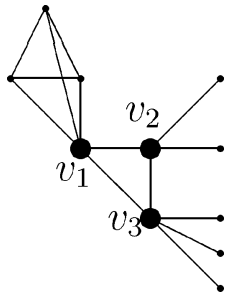

Fig. $1 . K_{3}(3 ; 2,3)$.

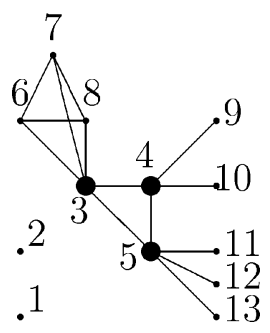

Fig. 2. Labeling $K_{13}-K_{3}(3 ; 2,3)$. $\left.\ldots, b_{m}\right)$, the MCSR graph is the so-called multi-star related (MSR) graph. A closed formula for counting the number of spanning trees of an MSR graph was derived in [3] for limited $m$ (in [6] for arbitrary $m$ ). However, the technique $[3,6]$ used in deriving the closed formulas for the number of spanning trees of an MSR graph cannot be used to derive the closed formula for the number of spanning trees of an MCSR graph straightforwardly.

Employing some new linear algebraic manipulations, this paper derives a closed formula for the number of spanning trees of an MCSR graph. The proposed results cover the previous results in $[1,2,4,5]$ and extend the results in $[3,6]$ to a larger graph class. In addition, we provide a general maximization theorem for the multi-star graph.

\section{Complement spanning tree matrix of an MCSR graph}

The complement spanning tree matrix (CSTM) [5] $C$ for a graph $G$ is defined by

$C_{i j}= \begin{cases}n-\bar{d}_{i}, & \text { if } i=j, \\ e_{i j}, & \text { if } i \neq j,\end{cases}$

where $\bar{d}_{i}$ is the degree of vertex $i$ in $\bar{G}$ and $e_{i j}$ is one if $(i, j)$ is in $E(\bar{G})$ and 0 otherwise.

From the result derived by Temperley [5], we have the following result.

Lemma 1 [5]. For the MCSR graph $G$, the number of spanning trees of $G, \tau(G)$, is equal to $|C| / n^{2}$, where $|C|$ represents the determinant of matrix $C$.

\section{Labeling the MCSR graph}

In this section, a method is presented for labeling the MCSR graph. Given the MCSR graph $G=K_{n}-$ $K_{m}\left(a_{1}, a_{2}, \ldots, a_{l} ; b_{1}, \ldots, b_{m-l}\right)$, we label the nodes in $V\left(K_{n}\right)-V\left(K_{m}\left(a_{1}, a_{2}, \ldots, a_{l} ; b_{1}, \ldots, b_{m-l}\right)\right)$ first. Next we label the roots of the $l$ complete graphs. Then we label the roots of the $m-l$ star graphs. Further, we label the nodes in the $i$ th complete graph except the root node for $i=1,2, \ldots, l$. Finally, we label the nodes in the $j$ th star graph except the root node for $j=1,2, \ldots, m-l$. Fig. 2 illustrates the labeling of the complement of $K_{13}-K_{3}(3 ; 2,3)$.

The CSTM of Fig. 2 is equal to

$C=\left(\begin{array}{ll}n I_{2} & \\ & D\end{array}\right)$,

where

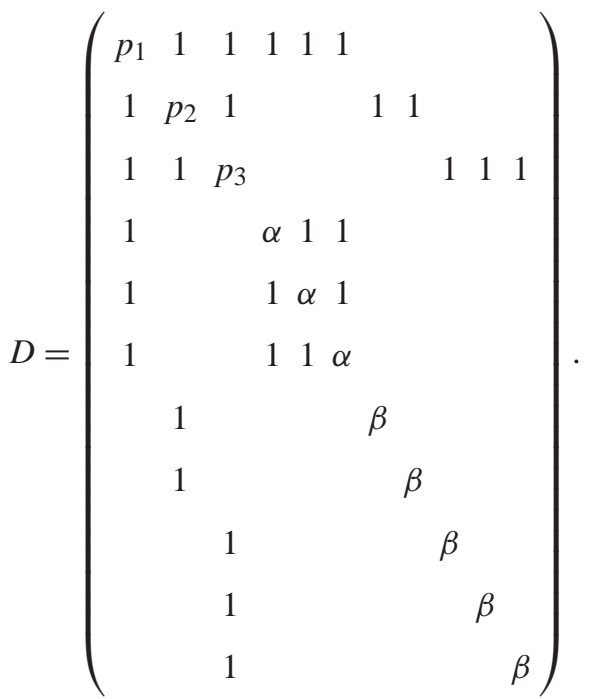

Here, $n=13, m=3, a_{1}=3, b_{1}=2, b_{2}=3, \alpha=$ $n-a_{1}, \beta=n-1, p_{1}=n-a_{1}-m+1, p_{2}=$ 
$n-b_{1}-m+1$, and $p_{3}=n-b_{2}-m+1$. We can represent the matrix $D$ in the following block-matrix form:

$D=\left(\begin{array}{cccc}P & \boldsymbol{e}_{1} \boldsymbol{f}_{1}^{\mathrm{T}} & \boldsymbol{e}_{2} \boldsymbol{f}_{2}^{\mathrm{T}} & \boldsymbol{e}_{3} \boldsymbol{f}_{3}^{\mathrm{T}} \\ \boldsymbol{f}_{1} \boldsymbol{e}_{1}^{\mathrm{T}} & A_{1} & & \\ \boldsymbol{f}_{2} \boldsymbol{e}_{2}^{\mathrm{T}} & & B_{1} & \\ \boldsymbol{f}_{3} \boldsymbol{e}_{3}^{\mathrm{T}} & & & B_{2}\end{array}\right)$,

where

$\boldsymbol{f}_{i}=(\underbrace{1,1, \ldots, 1}_{x_{i}})^{\mathrm{T}}$,

$\boldsymbol{e}_{i}=(\underbrace{0, \ldots, 0,1}_{i}, \underbrace{0, \ldots, 0}_{x_{i}-i})^{\mathrm{T}}$,

where $x_{i}=a_{i}$ for $1 \leqslant i \leqslant l ; x_{j}=b_{j}$ for $1 \leqslant j \leqslant m-l$,

$P=\left(\begin{array}{ccc}p_{1} & 1 & 1 \\ 1 & p_{2} & 1 \\ 1 & 1 & p_{3}\end{array}\right), \quad A_{1}=\left(\begin{array}{ccc}\alpha & 1 & 1 \\ 1 & \alpha & 1 \\ 1 & 1 & \alpha\end{array}\right)$,

$B_{1}=\beta I_{2}, \quad B_{2}=\beta I_{3}$.

\section{The closed formula}

For counting the number of spanning trees of an MCSR graph as mentioned in Lemma 1, the next theorem gives our closed formula which covers the closed formula in $[3,6]$ for counting the number of spanning trees of an MSR graph. The linear algebraic technique used in the proof is different from that used in [6].

Theorem 1. The number of spanning trees of an MCSR graph $G=K_{n}-K_{m}\left(a_{1}, a_{2}, \ldots, a_{l} ; b_{1}, \ldots\right.$, $\left.b_{m-l}\right)$ is equal to

$$
\begin{aligned}
\tau(G)= & n^{k-2}\left(1+\frac{a_{1}}{n-a_{1}-1}\right)\left(1+\frac{a_{2}}{n-a_{2}-1}\right) \cdots \\
& \cdot\left(1+\frac{a_{l}}{n-a_{l}-1}\right) \\
& \cdot\left(n-a_{1}-1\right)^{a_{1}}\left(n-a_{2}-1\right)^{a_{2}} \ldots \\
& \cdot\left(n-a_{l}-1\right)^{a_{l}}(n-1)^{b_{1}+\cdots+b_{m-l}}|Q|,
\end{aligned}
$$

where $|Q|=\left[1+1 /\left(q_{1}-1\right)+1 /\left(q_{2}-1\right)+\cdots+\right.$ $\left.1 /\left(q_{m}-1\right)\right]\left(q_{1}-1\right)\left(q_{2}-1\right) \cdots\left(q_{m}-1\right)$ where

$q_{i}=\left\{\begin{array}{c}n-a_{i}-m+1-\frac{a_{i}}{n-1} \\ \text { for } i=1,2, \ldots, l, \\ n-b_{i-l}-m+1-\frac{b_{i-l}}{n-1} \\ \quad \text { for } i=l+1, l+2, \ldots, m,\end{array}\right.$

and $k=n-m-a_{1}-a_{2}-\cdots-a_{l}-b_{1}-\cdots-b_{m-l}$.

Proof. As mentioned in Section 3, the CSTM of $G$ can be represented by

$C=\left(\begin{array}{ll}n I_{k} & \\ & D\end{array}\right)$,

where

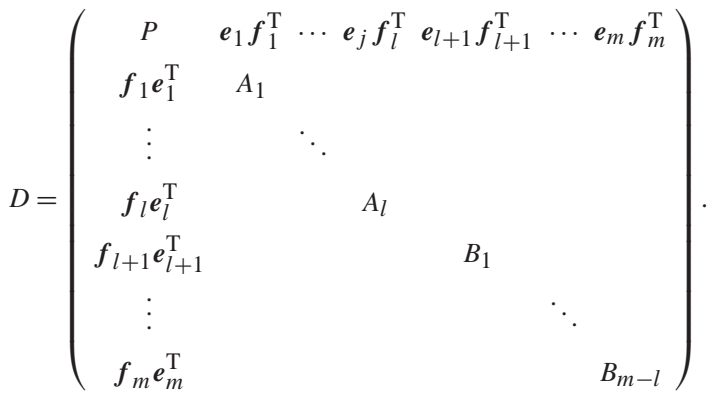

Here

$P=\left(\begin{array}{cccc}p_{1} & 1 & \cdots & 1 \\ 1 & p_{2} & \ddots & \vdots \\ \vdots & \ddots & \ddots & 1 \\ 1 & \cdots & 1 & p_{m}\end{array}\right)$,

$A_{i}=\left(\begin{array}{cccc}\alpha_{i} & 1 & \cdots & 1 \\ 1 & \alpha_{i} & \ddots & \vdots \\ \vdots & \ddots & \ddots & 1 \\ 1 & \cdots & 1 & \alpha_{i}\end{array}\right)_{a_{i} \times a_{i}}, \quad B_{i}=\beta I_{b_{i}}$,

where $\alpha_{i}=n-a_{i}$ for $1 \leqslant i \leqslant l, \beta=n-1, p_{i}=$ $n-a_{i}-m+1$ for $1 \leqslant i \leqslant l$, and $p_{i}=n-b_{i-l}-m+1$ for $l<i \leqslant m$. From Lemma 1 , the number of spanning trees of $G$ is $n^{k-2}|D|$. 
Let

$$
E=\left(\begin{array}{c}
I_{m} \\
c_{1} \boldsymbol{f}_{1} \boldsymbol{e}_{1}^{\mathrm{T}} \\
\vdots \\
c_{m} \boldsymbol{f}_{m} \boldsymbol{e}_{m}^{\mathrm{T}}
\end{array}\right),
$$

where $c_{1}, c_{2}, \ldots, c_{m}$ are to be determined. Since

$$
D E=\left(\begin{array}{c}
P+c_{1} \boldsymbol{e}_{1} \boldsymbol{f}_{1}^{\mathrm{T}} \boldsymbol{f}_{1} \boldsymbol{e}_{1}^{\mathrm{T}}+\cdots+c_{m} \boldsymbol{e}_{m} \boldsymbol{f}_{m}^{\mathrm{T}} \boldsymbol{f}_{m} \boldsymbol{e}_{m}^{\mathrm{T}} \\
\boldsymbol{f}_{1} \boldsymbol{e}_{1}^{\mathrm{T}}+c_{1} A_{1} \boldsymbol{f}_{1} \boldsymbol{e}_{1}^{\mathrm{T}} \\
\vdots \\
\boldsymbol{f}_{l} \boldsymbol{e}_{l}^{\mathrm{T}}+c_{l} A_{l} \boldsymbol{f}_{l} \boldsymbol{e}_{l}^{\mathrm{T}} \\
\boldsymbol{f}_{l+1} \boldsymbol{e}_{l+1}^{\mathrm{T}}+c_{l+1} B_{1} \boldsymbol{f}_{l+1} \boldsymbol{e}_{l+1}^{\mathrm{T}} \\
\vdots \\
\boldsymbol{f}_{m} \boldsymbol{e}_{m}^{\mathrm{T}}+c_{m} B_{m-l} \boldsymbol{f}_{m} \boldsymbol{e}_{m}^{\mathrm{T}}
\end{array}\right),
$$

we want to determine $c_{1}, c_{2}, \ldots, c_{m}$ such that the entries of $D E$ are all zero except the first entry. Therefore, we have that

$$
\begin{array}{ll}
A_{i} \boldsymbol{f}_{i} \boldsymbol{e}_{i}^{\mathrm{T}}=(n-1) \boldsymbol{f}_{i} \boldsymbol{e}_{i}^{\mathrm{T}} & \text { for } i=1,2, \ldots, l, \\
B_{i-j} \boldsymbol{f}_{i} \boldsymbol{e}_{i}^{\mathrm{T}}=(n-1) \boldsymbol{f}_{i} \boldsymbol{e}_{i}^{\mathrm{T}} & \text { for } i=l+1, l+2, \ldots, m .
\end{array}
$$

After setting

$c_{i}=-\frac{1}{n-1} \quad$ for $i=1,2, \ldots, m$,

we have

$D E=\left(\begin{array}{c}Q \\ 0 \\ \vdots \\ 0\end{array}\right)$,

where

$$
\begin{aligned}
Q & =P+c_{1} \boldsymbol{e}_{1} \boldsymbol{f}_{1}^{\mathrm{T}} \boldsymbol{f}_{1}^{\mathrm{T}}+\cdots+c_{m} \boldsymbol{e}_{m} \boldsymbol{f}_{m}^{\mathrm{T}} \boldsymbol{f}_{m}^{\mathrm{T}} \\
& =\left(\begin{array}{cccc}
q_{1} & 1 & \cdots & 1 \\
1 & q_{2} & \ddots & \vdots \\
\vdots & \ddots & \ddots & 1 \\
1 & \cdots & 1 & q_{m}
\end{array}\right)
\end{aligned}
$$

and

$$
q_{i}= \begin{cases}p_{i}-\frac{a_{i}}{n-1} & \text { for } i=1,2, \ldots, l, \\ p_{i}-\frac{b_{i-l}}{n-1} & \text { for } i=l+1, l+2, \ldots, m .\end{cases}
$$

Further, we let

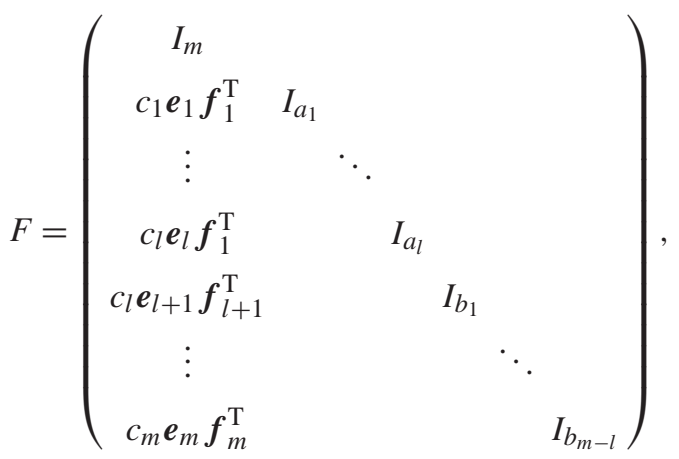

then we have

$$
D F=\left(\begin{array}{cccccc}
Q \boldsymbol{e}_{1} \boldsymbol{f}_{1}^{\mathrm{T}} & \cdots & \boldsymbol{e}_{l} \boldsymbol{f}_{l}^{\mathrm{T}} & \boldsymbol{e}_{l+1} \boldsymbol{f}_{l+1}^{\mathrm{T}} \cdots & \boldsymbol{e}_{m} \boldsymbol{f}_{m}^{\mathrm{T}} \\
A_{1} & & & & & \\
& \ddots & & & & \\
& & A_{l} & & & \\
& & & B_{1} & & \\
& & & & \ddots & \\
& & & & & B_{m-l}
\end{array}\right) .
$$

Since the matrix $D F$ is a block upper triangular matrix, consequently, it yields

$$
\begin{aligned}
\operatorname{det}(D)= & \operatorname{det}(D F)=\operatorname{det}(D) \operatorname{det}(F) \\
= & \operatorname{det}(Q) \operatorname{det}\left(A_{1}\right) \cdots \operatorname{det}\left(A_{l}\right) \\
& \cdot \operatorname{det}\left(B_{1}\right) \cdots \operatorname{det}\left(B_{m-l}\right) .
\end{aligned}
$$

It is observed that the matrix $Q$ has diagonal elements $q_{1}, q_{2}, \ldots, q_{m}$ and has ones on the offdiagonals. Following the eliminating technique in [6], we border $Q$ by adding a new first row and column. All the entries in the new row are ones, but the entries in the new column which are not in the new row are zeros. This row and column augmentation will preserve the same determinant as $Q$.

Subtract the new row from each of the other rows. The diagonal entries of $Q$ in the old row indexed $i$ are now $q_{i}-1$. The first column consists of all 
-1 's except for the one in the first row. The first row consists of all ones. Excepting the diagonal elements, the remaining elements of the matrix are zero. Now add to the first column $1 /\left(q_{i}-1\right)$ times each of the other columns to zero out the off-diagonal entries in this column. The entry in the first row and column becomes $1+1 /\left(q_{1}-1\right)+1 /\left(q_{2}-1\right)+\cdots+1 /\left(q_{m}-\right.$ $1)$. Thus, the matrix $Q$ becomes upper triangular, so we have

$$
\begin{aligned}
\operatorname{det}(Q)= & {\left[1+\frac{1}{q_{1}-1}+\frac{1}{q_{2}-1}+\cdots+\frac{1}{q_{m}-1}\right] } \\
& \cdot\left(q_{1}-1\right)\left(q_{2}-1\right) \cdots\left(q_{m}-1\right) .
\end{aligned}
$$

By the same argument, the matrix $A_{i}$ has diagonal elements $\alpha_{i}, \alpha_{i}, \ldots, \alpha_{i}$ and has ones on the offdiagonals. It can be verified that

$$
\begin{aligned}
\operatorname{det}\left(A_{i}\right) & =\left(1+\frac{a_{i}}{\alpha_{i}-1}\right)\left(\alpha_{i}-1\right)^{a_{i}} \\
& =\left(1+\frac{a_{i}}{n-a_{i}-1}\right)\left(n-a_{i}-1\right)^{a_{i}}
\end{aligned}
$$

for $1 \leqslant i \leqslant l$.

In addition, the matrix $B_{j}$ has diagonal elements $\beta, \beta$, $\ldots, \beta$ and has zeros on the off-diagonals, so $\operatorname{det}\left(B_{j}\right)=\beta^{b_{j}}=(n-1)^{b_{j}}$ for $1 \leqslant j \leqslant m-l$.

Consequently, the number of spanning trees of $G$, $\tau(G)$, is equal to $n^{k-2}|D|$. Here $|D|$ is equal to

$$
\begin{gathered}
\left(1+\frac{a_{1}}{n-a_{1}-1}\right)\left(1+\frac{a_{2}}{n-a_{2}-1}\right) \cdots \\
\cdot\left(1+\frac{a_{l}}{n-a_{l}-1}\right) \\
\cdot\left(n-a_{1}-1\right)^{a_{1}}\left(n-a_{2}-1\right)^{a_{2}} \ldots \\
\cdot\left(n-a_{l}-1\right)^{a_{l}}(n-1)^{b_{1}+\cdots+b_{m-l}}|Q|,
\end{gathered}
$$

where $|Q|=\left[1+1 /\left(q_{1}-1\right)+1 /\left(q_{2}-1\right)+\cdots+\right.$ $\left.1 /\left(q_{m}-1\right)\right]\left(q_{1}-1\right)\left(q_{2}-1\right) \cdots\left(q_{m}-1\right)$. Therefore,

$$
\begin{aligned}
\tau(G)= & n^{k-2}\left(1+\frac{a_{1}}{n-a_{1}-1}\right)\left(1+\frac{a_{2}}{n-a_{2}-1}\right) \cdots \\
& \cdot\left(1+\frac{a_{l}}{n-a_{l}-1}\right) \\
& \cdot\left(n-a_{1}-1\right)^{a_{1}}\left(n-a_{2}-1\right)^{a_{2}} \ldots \\
& \cdot\left(n-a_{l}-1\right)^{a_{l}}(n-1)^{b_{1}+\cdots+b_{m-l}}|Q|,
\end{aligned}
$$

where $|Q|=\left[1+1 /\left(q_{1}-1\right)+1 /\left(q_{2}-1\right)+\cdots+\right.$ $\left.1 /\left(q_{m}-1\right)\right]\left(q_{1}-1\right)\left(q_{2}-1\right) \cdots\left(q_{m}-1\right)$. We complete the proof.

\section{Maximization theorem for a multi-star graph}

In this section, a rather general maximization theorem is provided for the multi-star graph $G_{n}=K_{n}-$ $K_{m}\left(b_{1}, b_{2}, \ldots, b_{m}\right)$. For the same graph, in [3], a maximization theorem was provided only for $m=2,3$, and 4 , respectively.

From Theorem 1, considering the multi-star graph, we have

Corollary 1. The number of spanning trees of an MSR graph $G=K_{n}-K_{m}\left(b_{1}, \ldots, b_{m}\right)$ is equal to $\tau(G)=$ $n^{k-2}(n-1)^{b_{1}+\cdots+b_{m}}|Q|$, where $|Q|=\left[1+1 /\left(q_{1}-1\right)\right.$ $\left.+1 /\left(q_{2}-1\right)+\cdots+1 /\left(q_{m}-1\right)\right]\left(q_{1}-1\right)\left(q_{2}-1\right)$ $\cdots\left(q_{m}-1\right)$ where

$q_{i}=n-b_{i}-m+1-\frac{b_{i}}{n-1} \quad$ for $i=1,2, \ldots, m$

and $k=n-m-b_{1}-\cdots-b_{m}$.

Since $q_{i}=n-b_{i}-m+1-b_{i} /(n-1)$ and $b_{i} \leqslant$ $\sum_{k=1}^{m} b_{k} \leqslant n-m$, we have

$$
\begin{aligned}
q_{i} & =n-m+1-\frac{n b_{i}}{n-1} \\
& \geqslant n-m+1-\frac{n(n-m)}{n-1} \\
& =\frac{(n-m+1) n-(n-m+1)-n(n-m)}{n-1} \\
& =\frac{n-(n-m+1)}{n-1} \\
& =\frac{m-1}{n-1} \\
& >0 .
\end{aligned}
$$

We thus have $q_{i}>0$. For $i \neq j$, it yields to

$$
\begin{aligned}
q_{i}+q_{j} & =2(n-m+1)-\frac{n\left(b_{i}+b_{j}\right)}{n-1} \\
& \geqslant 2(n-m+1)-\frac{n(n-m)}{n-1} \\
& =(n-m+1)+(n-m+1)-\frac{n(n-m)}{n-1} \\
& >(n-m+1) .
\end{aligned}
$$

Based on a reasonable assumption, there exists a positive $b_{i}$. That is, we have $n-m \geqslant \sum_{k=1}^{m} b_{k}>0$ and it implies that $q_{i}+q_{j}>2$. 
Let

$$
\begin{aligned}
& f_{m}\left(b_{1}, b_{2}, \ldots, b_{m}\right) \\
& \quad=\left(1+\frac{1}{p_{1}}+\frac{1}{p_{2}}+\cdots+\frac{1}{p_{m}}\right)\left(p_{1} p_{2} \cdots p_{m}\right),
\end{aligned}
$$

where

$p_{k}=q_{k}-1=n-m-\frac{n b_{k}}{n-1}>-1 ;$

the nonnegative integers $b_{1}, b_{2}, \ldots, b_{m}$ satisfy the constraint $b_{1}+b_{2}+\cdots+b_{m}=L$, where $L$ is a constant. From $p_{i}+p_{j}=q_{i}-1+q_{j}-1>0$ for all $i \neq j$, there is at most one negative $q_{k}$ for $1 \leqslant k \leqslant m$. We now want to find the maximum of the function $f_{m}\left(b_{1}, b_{2}, \ldots, b_{m}\right)$.

Suppose when $\left(b_{1}, b_{2}, \ldots, b_{m}\right)=\left(\hat{b}_{1}, \hat{b}_{2}, \ldots, \hat{b}_{m}\right)$, we make function $f_{m}\left(b_{1}, b_{2}, \ldots, b_{m}\right)$ maximal. Let

$\hat{p}_{k}=n-m-\frac{n \hat{b}_{k}}{n-1} \quad$ for $k=1,2, \ldots, m$.

The maximization theorem for the multi-star graph is proved by the technique of contradiction. Suppose $\hat{b}_{i}>\hat{b}_{j}+1$. We consider two cases. In case 1 , if there exists an $l$ such that $\hat{p}_{l}<0$, then we have $\hat{b}_{l} \geqslant \hat{b}_{i}$, i.e., $\hat{b}_{l}>\hat{b}_{j}+1$. For all $k \neq l, j$, we thus have $\hat{p}_{k}>0$. In case 2 , for all $k \neq i, j$, we have $\hat{p}_{k}>0$.

By the symmetric property of $f_{m}\left(b_{1}, b_{2}, \ldots, b_{m}\right)$, for case 1 , we assume $l=1$ and $j=2$ for convenience and for case 2, we assume $i=1$ and $j=2$. Hence we have $\hat{b}_{1}>\hat{b}_{2}+1$ and for all $k \geqslant 3$, we have $\hat{p}_{k}>0$. The maximum of $f_{m}\left(b_{1}, b_{2}, \ldots, b_{m}\right)$ is given by

$$
\begin{aligned}
f_{m}\left(\hat{b}_{1}, \hat{b}_{2}, \ldots, \hat{b}_{m}\right)= & f_{m-2}\left(\hat{b}_{3}, \hat{b}_{4}, \ldots, \hat{b}_{m}\right)\left(\hat{p}_{1} \hat{p}_{2}\right) \\
& +\left(\hat{p}_{1}+\hat{p}_{2}\right)\left(\hat{p}_{3} \hat{p}_{4} \cdots \hat{p}_{m}\right) .
\end{aligned}
$$

Since each $\hat{p}_{k}>0$ for $k=3,4, \ldots, m$, we have $f_{m-2}\left(\hat{p}_{3}, \hat{p}_{4}, \ldots, \hat{p}_{m}\right)>0$.

Now let

$$
\begin{aligned}
& \hat{p}_{1}^{\prime}=n-m-\frac{n\left(b_{1}-1\right)}{n} \text { and } \\
& \hat{p}_{2}^{\prime}=n-m-\frac{n\left(b_{2}+1\right)}{n},
\end{aligned}
$$

then we have $\hat{p}_{1}^{\prime}+\hat{p}_{2}^{\prime}=\hat{p}_{1}+\hat{p}_{2}$ and

$$
\begin{aligned}
\hat{p}_{1}^{\prime} \hat{p}_{2}^{\prime} & =\frac{1}{4}\left[\left(\hat{p}_{1}^{\prime}+\hat{p}_{2}^{\prime}\right)^{2}-\left(\hat{p}_{1}^{\prime}-\hat{p}_{2}^{\prime}\right)^{2}\right] \\
& >\frac{1}{4}\left[\left(\hat{p}_{1}+\hat{p}_{2}\right)^{2}-\left(\hat{p}_{1}-\hat{p}_{2}\right)^{2}\right] \\
& =\hat{p}_{1} \hat{p}_{2} .
\end{aligned}
$$

From the above inequality, we have

$$
\begin{aligned}
f_{m} & \left(\hat{b}_{1}-1, \hat{b}_{2}+1, \ldots, \hat{b}_{m}\right) \\
= & f_{m-2}\left(\hat{b}_{3}, \hat{b}_{4}, \ldots, \hat{b}_{m}\right)\left(\hat{p}_{1}^{\prime} \hat{p}_{2}^{\prime}\right) \\
& +\left(\hat{p}_{1}^{\prime}+\hat{p}_{2}^{\prime}\right)\left(\hat{p}_{3} \hat{p}_{4} \cdots \hat{p}_{m}\right) \\
> & f_{m-2}\left(\hat{b}_{3}, \hat{b}_{4}, \ldots, \hat{b}_{m}\right)\left(\hat{p}_{1} \hat{p}_{2}\right) \\
& +\left(\hat{p}_{1}+\hat{p}_{2}\right)\left(\hat{p}_{3} \hat{p}_{4} \cdots \hat{p}_{m}\right) \\
= & f_{m}\left(\hat{b}_{1}, \hat{b}_{2}, \ldots, \hat{b}_{m}\right) .
\end{aligned}
$$

It is a contradiction. That is, the assumption $\hat{b}_{1}>$ $\hat{b}_{2}+1$ is false. On the contrary, we must have $\hat{b}_{1} \leqslant$ $\hat{b}_{2}+1$. Generally, considering all pair of $i$ and $j$ and from Corollary 1 , we have the following maximization theorem for the multi-star graph.

Theorem 2. The number of spanning trees of an MSR graph $G=K_{n}-K_{m}\left(b_{1}, \ldots, b_{m}\right)$ is maximal when the difference between $b_{i}$ and $b_{j}$ for $1 \leqslant i, j \leqslant m$ is at most one.

\section{Conclusion}

Graphs are often used to model network problems. For example, we often use star graphs (like MSR), complete graphs, or hybrid graphs (like MCSR) to model connection patterns in a network. Among these graphs, finding a spanning tree with some specific properties, such as minimum weight and minimum label, is a well-known technique to solve the network routing problem. Since the number of spanning trees in a graph could reveal the complexity of the corresponding combinatorial configuration, deriving a closed form of the number of spanning trees is indeed a research problem. Since the CSTM form of the MCSR graph is much more complicated than that of the MSR graph, deriving the corresponding closed form of the MCSR is much harder than that of the MSR graph.

We have presented how to derive the closed form for the number of spanning trees of an MCSR graph $G=$ $K_{n}-K_{m}\left(a_{1}, a_{2}, \ldots, a_{l} ; b_{1}, \ldots, b_{m-l}\right)$ for arbitrary $m$. The main contribution of this paper is that the derived closed formula extends that of the graph class discussed in the previous results [3,6] of the MSR graph to a larger MCSR graph class. Another minor contribution of this paper is that a general 
maximization theorem is provided for the multi-star graph.

\section{Acknowledgement}

The authors are indebted to the anonymous referees and editor Professor Lane A. Hemaspaandra for their valuable suggestions that lead to the improved version of the paper.

\section{References}

[1] C. Berge, Graphs and Hypergraphs, North-Holland, Amsterdam, 1973.
[2] W. Moon, Enumerating labeled trees, in: F. Harary (Ed.), Graph Theory and Theoretical Physics, Academic Press, London, 1967, pp. 261-271.

[3] S.D. Nikolopoulos, P. Rondogiannis, On the number of spanning trees of multi-star related graphs, Inform. Process. Lett. 65 (1998) 183-188.

[4] P.V. O'Neil, The number of trees in a certain network, Notices Amer. Math. Soc. 10 (1963) 569

[5] H.N.V. Temperley, On the mutual cancellation of cluster integrals in Mayer's fugacity series, Proc. Phys. Soc. 83 (1964) 3 16.

[6] W.M. Yan, W. Myrvold, K.L. Chung, A formula for the number of spanning trees of a multi-star related graph, Inform. Process. Lett. 68 (1998) 295-298. 